\title{
Intelligent Fault Classification of Rolling Bearing at Variable Speed Based on Reconstructed Phase Space
}

\author{
Weigang Wen \\ School of Mechanical, Electronic and Control Engineering, Beijing Jiaotong University \\ Beijing 100044, China \\ Weidong Cheng \\ School of Mechanical, Electronic and Control Engineering, Beijing Jiaotong University \\ Beijing 100044, China \\ E-mail:wgwen@bjtu.edu.cn
}

\begin{abstract}
Rolling bearing is a common mechanical part which is subject to be damaged. It is important to monitor the condition of bearing. An effective mean is to extract faulty features of bearing from the vibration signal. In this paper, a method is introduced to realize intelligent classification of bearing state. The vibration signal is reconstructed into phase space by estimating the time delay and embedded dimension of time series. After reconstruction, fault classification is accomplished through normalized principal component analysis. It is testified that this method is effective for classifying fault of bearing by experiment and data analysis.
\end{abstract}

Keywords: Fault classification; Time delay; embedded dimension; phase space reconstruction; principal components analysis.

\section{Introduction}

The rolling bearings are support elements in many kinds of rotating machinery. Many machines are working at variable rotating speed. The bearing faults always cause the structural damage of machine. The measure to identify the rolling bearing fault and judge its fault type intelligently is crucial in state monitoring of machine. The common way to diagnose the rolling bearing faults is the method of vibration signal analysis. Many techniques have been proposed to obtain features of vibration signals for fault diagnosis. A lot of researches focus on extracting the fault information from the vibration signal by time and frequency domain signal processing techniques. These methods include FFT, Envelope analysis, EMD, wavelet and wavelet packet method, ${ }^{1-4}$ etc. Especially, the order tracking technique was proposed to solving the rolling bearing fault diagnosis at variable shaft rotational speed., 6

But because of instantaneous variations in rolling ball movement, friction, damping and machine load, the rolling bearing is often characterized by non-linear behaviors. The non-linear analysis can provide a good alternative method to draw fault features from the vibration signals. A lot of non-linear methods, such as correlation dimension, Lyapunov exponent and approximate entropy, have been investigated. ${ }^{7,8}$ The results have shown that non-linear fault diagnosis is an effective method. But in real application, it is quite a difficult problem on how to decide the threshold of the non-linear features. Moreover the non-linear features are not only sensitive to fault, especially when the state of the bearing is 
non-stationary, such as the variable rotating speed, the changing load, and the noise in the sampled signal, etc.

This paper mainly discusses the intelligent fault diagnosis of rolling bearing at variable speed. The paper is organized as follows: Section 2 addresses the main principles of methods including method of reconstructed phase space (RPS) and the normalized principal component analysis (PCA). The section involves the whole scheme of fault diagnosis. In Section 3, the vibration signal of different bearing status at various speeds is collected and analyzed using these methods. Moreover, selection of time delay, embedding dimension are discussed in this section. In Section4, the conclusions are presented finally.

\section{Main Principles and Methods}

\subsection{Reconstructing phase space}

In order to extract the non-linear feature of vibration signal, the dynamic system embedded in time series need to be reconstructed. The dynamics can be built on the time series by reconstructing phase space which is same as original system according to Takens's theorem. RPS method is very useful and has been widely applied to a variety of nonlinear signals processing applications. The basic idea of the RPS is that a scalar time series $x(\mathrm{t})$ may be used to construct a time sequence of vectors that is equivalent to the original dynamics from a topological point of view. If the proper embedded dimension $m$ and time delay $\tau$ can be chosen, based on $N$ sample time series $x=\{x(1)$, $x(2), \ldots, x(N-1), x(N)\}$, the phase space can be obtained:

$$
X(t)=[x(t), x(t+\tau), \ldots, x(t+(m-1) \tau)]
$$

Where the vector of $X(t)$ is a point in the RPS, $t=1,2, \ldots, N-(m-1) \tau$. The sufficient condition for topological equivalence of RPS with original system is that $m$ is greater than twice the dimension of the original dynamic system. When the embedded dimension is not known, as is the case for most real systems, there are many methods to calculate $m$ and $\tau$. C-C method is adopted in this paper. The advantages of this method are that its robustness to noise for vibration signal, the value of embedded dimension $m$ and time delay $\tau$ can be obtained simultaneously, there is no need to set the threshold value manually, and small sample data is needed. The principle of $\mathrm{C}$-C method is described as follows. ${ }^{9}$

The $N$ sample time series $x$ is divided into $t$ time series which are disjoint:

$$
\begin{aligned}
& \{x(1), x(t+1), x(2 t+1), \ldots \ldots\} \\
& \{x(2), x(t+2), x(2 t+2), \ldots \ldots\} \\
& \ldots \ldots \ldots . . . \\
& \{x(t), x(2 t), x(3 t), \ldots \ldots\}
\end{aligned}
$$

$S(m, N, r, t)$ of each time series is defined as:

$$
\begin{gathered}
S(m, N, r, t)=\frac{1}{t} \sum_{s=1}^{t}\left[C_{s}\left(m, \frac{N}{t}, r, t\right)-\right. \\
\left.C_{S}\left(1, \frac{N}{t}, r, t\right)\right]
\end{gathered}
$$

Where $C(m, N, r, t)$ is the correlation integral of time series:

$C(m, N, r, t)=\frac{2}{M(M-1)} \sum_{1 \leq i \leq j \leq M} \theta\left(r-d_{i j}\right)$

Where $\theta(x)=\left\{\begin{array}{l}0, x<0 \\ 1, x \geq 0\end{array} \quad d_{i j}=\left\|X_{i}-X_{j}\right\|\right.$,

$r>0, \quad M=N-(m-1) \tau$, and \|\| is infinite norm. When $N \rightarrow \infty$, there is:

$S(m, r, t)=\frac{1}{t} \sum_{s=1}^{t}\left[C_{s}(m, r, t)-C_{s}(1, r, t)\right]$

The maximum difference of $\mathrm{S}$ is defined as:

$\Delta S(m, t)=\max \{S(m, r, t)\}-\min \{S(m, r, t)\}$

The local maximum time corresponds to the zero of $S(m, r, t)$ and the minimum of $\Delta S(m, t)$. The time delay $\tau$ is the first local maximum time. The statistics is defined as:

$$
S_{\text {cor }}(t)=\Delta \bar{S}(t)+|\bar{S}(t)|
$$

Where $\Delta \bar{S}(t)$ and $\bar{S}(t)$ are the averages of $\Delta S(t)$ and $S(t)$. The time window $\tau_{w}=m \tau$ corresponds to the minimum of $S_{\text {cor }}(t)$. In practical application, $\frac{\sigma}{2} \leq r \leq 2 \sigma, \sigma$ is the standard deviation of time series, $N \geq 500$ and $N=3000$ is applied for vibration signal time series.

\subsection{Normalized principal component analysis}

The fault of rolling bearing causes the change of dynamics of mechanical system. Phase trajectory of dynamical systems could be obtained by 
phase spacer construction of vibration signal time series. The different features of signals generated by topologically different systems could be drawn out through RPS. In comparison with the eigen-value, such as correlation dimension, Kolmogorov entropy and Lyapunov exponents, the phase trajectory could describe completely the reconstructed dynamical system, especially for the pseudo-random time series of rolling bearing vibration signal. In this paper, the principal components of RPS are used to describe the characteristics of multi dimension phase trajectory in phase space. ${ }^{10}$ The advantage of this method is that it is applicable to time series contaminated by noise which really exist in mechanical signal.

From the $N$ sample time series and the RPS $X(t)$, the phase trajectory of the dynamics system can be drawn by the matrix $Y$ :

$$
Y_{n \times m}=\frac{1}{n^{1 / 2}}\left[\begin{array}{c}
X(1) \\
X(2) \\
\vdots \\
X(n)
\end{array}\right]
$$

Where $n=N-(m-1) \tau$. And the con-variance matrix $A$ is:

$$
A_{m \times m}=Y_{n \times m}^{T} Y_{n \times m}
$$

Then the eigen-values $\lambda_{i}(i=1,2, \cdots m)$ and the eigen-vectors $U_{i}(i=1,2, \cdots m)$ of matrix $A_{m \times m}$ can be calculated. $\lambda_{i}$ and $U_{i}$ are the principal components. The eigen-values are listed in descending order:

$$
\lambda_{1} \geq \lambda_{2} \geq \cdots \geq \lambda_{m}
$$

The principal component spectrum can be drawn from $\lambda_{i}$. The principal component spectrum of noise is horizontal. The characteristics of the dynamics system can be extracted from the maximal principal components excluding the horizontal minimal components. The scale of vibration signal is different at various rotating speed. In order to classify the fault intelligently, the principal components must be normalized. The sum of $\lambda_{i}$ is:

$$
\gamma=\sum_{i=1}^{m} \lambda_{i}
$$

The normalized principal components are:

$$
\lambda_{i}^{n}=\frac{\lambda_{i}}{\gamma}
$$

$\lambda_{1}^{n}, \lambda_{2}^{n}, \cdots$ can be used as classification components. So the normalized principal components analysis can be utilized for the classification of fault in rolling bearing with various speeds, loads and noise.

\section{Experiments and Discussions}

In order to testify the validity of method proposed above, experimental data were collected from rolling bearing of an experimental mechanical system. The SKF rolling bearings was applied to support the rotating shaft. An accelerometer was mounted on the housing near the rolling bearing to acquire the vibration signals of the bearing. Single point faults were introduced to the test bearings using electro-discharge machining. The vibration signal was sampled from bearing at various speeds. The class of data corresponds to the bearing conditions respectively: normal bearing, and outer race fault, etc.

The vibration signal time series of bearing with different faults and various rotating speeds are shown in Fig. 1. The sample rate is $24 \mathrm{k} \mathrm{Hz}$. (a) is the normal bearing at 1000rpm, (b) is the normal bearing at 2500rpm, (c) is the normal bearing at $4500 \mathrm{rpm}$, (d) is the outer race faulty bearing at 2000rpm, (e) is the outer race faulty bearing at 3000rpm, (f) is the outer race faulty bearing at 4500rpm.
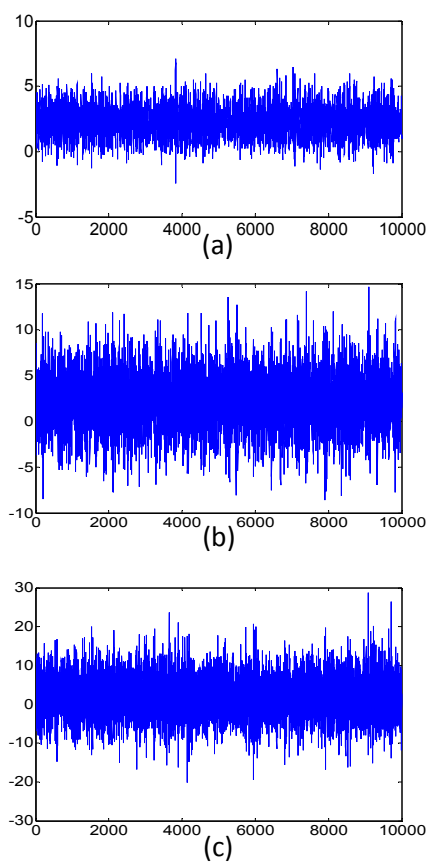

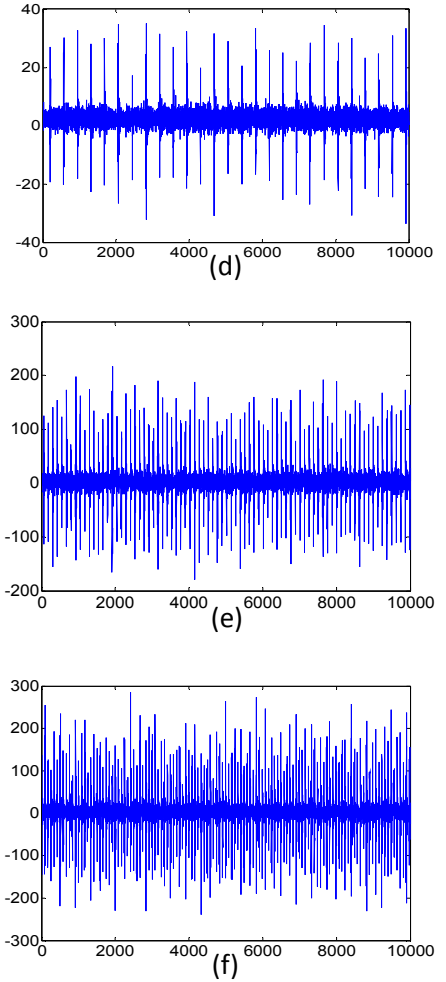

Fig.1. The vibration signal time series of bearing with different faults and various rotating speeds

At first, the phase space is reconstructed. The embedded dimension $m$ and time delay $\tau$ are obtained simultaneously through C-C method. The result of C-C method is shown in Fig. 2. Here the structure of RPS is that embedded dimension $m$ is set to 26 and time delay $\tau$ is set to 6 .

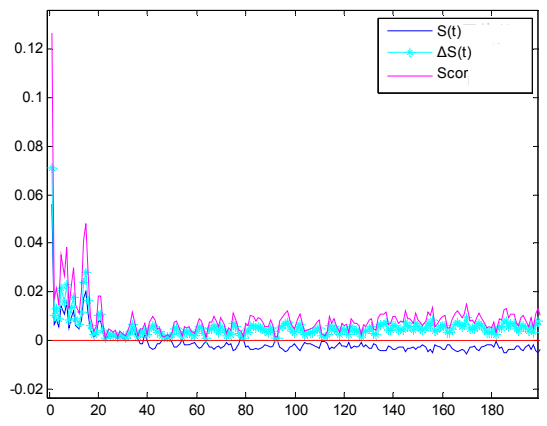

Fig.2. The result of C-C method

Then the principal components can be calculated. The principal component spectrum of vibration signal is shown in Fig. 3. (a) is the PCS of normal bearing at 1000rpm, (b) is the PCS of normal bearing at 2500rpm, (c) is the PCS of normal bearing at $4500 \mathrm{rpm}$, (d) is the PCS of outer race faulty bearing at 2000rpm, (e) is the PCS of outer race faulty bearing at 3000rpm, (f) is the PCS of outer race faulty bearing at 4500rpm. The similarity of same bearing at various speeds is shown in the PCS, shown in (a)-(c) normal bearing and (d)-(f) faulty bearing. And the components following the second component increase along with increasing speed. The difference between the normal bearing and faulty bearing is clear. The first component is far larger than the other components in the PCS of normal bearing. And the second component is almost equal to the first component in the PCS of outer race faulty bearing. The features of the dynamics system can be drawn from the maximal principal components.

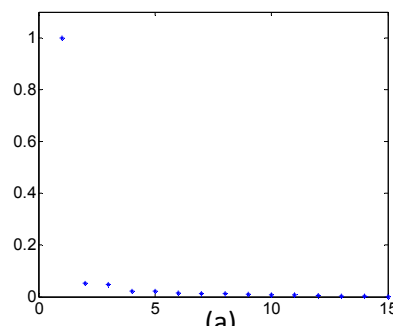

(a)
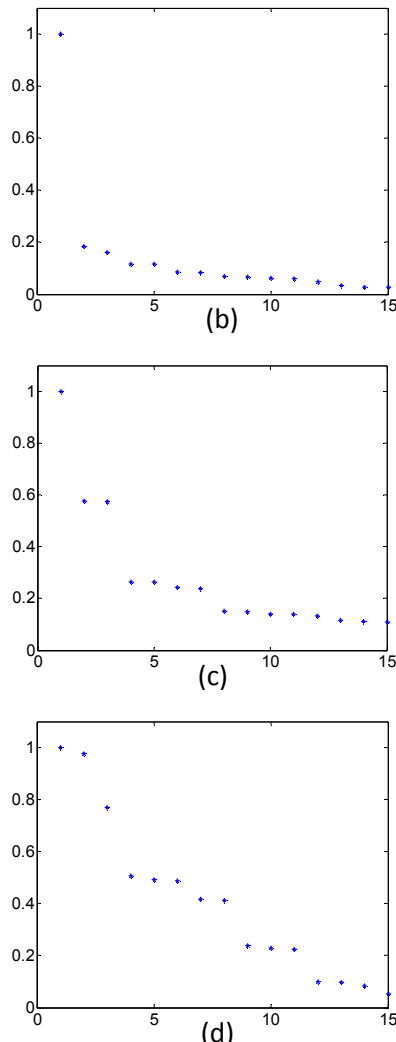

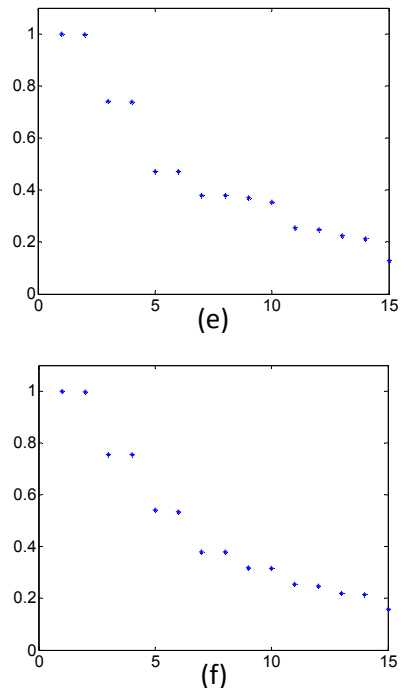

Fig.3. The principal component spectrum

12 samples of normal and fault bearing at different speed are tested respectively. After normalization, principal component analysis method is implemented in 3 dimension space. The plot of the first three principal components (PCs) of the clustering results is shown in Fig. 4. Almost all samples are correctly classified to the corresponding clusters by the method.

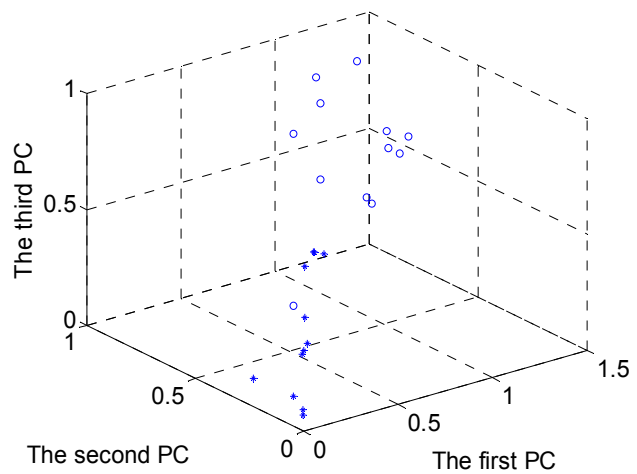

Fig. 4. Scatter plot of principal components for clustering result

\section{Conclusions}

A method that integrates RPS and NPCA is presented in this paper to implement detection and classification of bearing faults. Reconstruction of vibration signal in phase space is made through $\mathrm{C}-\mathrm{C}$ method. Then normalized principal components analysis is applied to extract the features from the phase trajectory of faulty bearing. It is proved that this method is effective for classifying fault of bearing by experiment and data analysis. But in the experiment, the classification of the faulty bearing through principal components is difficult at the speed lower than about 1000rpm. And vibration signals adopted here are only single point fault. So the work in the next step could be focused on classification for the more variable rotating speed and the multiple points bearing fault.

\section{Acknowledgements}

This project is supported by the Fundamental Research Funds for the Central Universities (2011JBM093) and National Natural Science Foundation of China (51275030).

\section{References}

1. H. R. Martin, F. Honarvar, Application of statistical moments to bearing failure detection, Applied Acoustics 44 (1995) 67-77.

2. R.Jones, Enveloping for bearing analysis, Sound and Vibration 30 (1996) 10-15.

3. H.Ocak, K.A. Loparo, F.M.Discenzo, On line tracking of bearing wear using wavelet packet decomposition and probabilistic modeling: A method for bearing prognostics, Journal of Sound and Vibration 302 (2007) 951-961.

4. R.B. Randall, J. Antoni, Rolling element bearing diagnostics-a tutorial, Mech. Syst. Signal Process. 25 (2011) 485-520.

5. K.R. Fyfe, E.D.S. Munck, Analysis of computed order tracking, Mech. Syst. Signal Process. 11 (1997) 187-205.

6. P. Borghesani, R. Ricci, S. Chatterton, P. Pennacchi, A new procedure for using envelope analysis for rolling element bearing diagnostics in variable operating conditions, Mech. Syst. Signal Process. 38 (2013) 23-35.

7. GuoFeng Wang, YuBo Li, ZhiGao Luo, Fault classification of rolling bearing based on reconstructed phase space and Gaussian mixture model, Journal of Sound and Vibration 323 (2009) 1077-1089.

8. Min $\mathrm{Li}$, Jinwu $\mathrm{Xu}$, Jianhong Yang, Multiple manifolds analysis and its application to fault diagnosis, Mechanical Systems and Signal Processing 23 (2009) 2500-2509.

9. Kim H. S., Eykholt R., Salas J. D., Nonlinear dynamics, delay times, and embedding windows,

Published by Atlantis Press

Copyright: the authors 
Physica D: Nonlinear Phenomena 127 (1999) 48-60.

10. Q. He, R. Yan, F. Kong, and R. Du, Machine condition monitoring using principal component representations, Mechanical Systems and Signal Processing 23 (2) (2009) 446-466. 